\title{
MAGNETIC FIELDS IN OUR GALAXY: HOW MUCH DO WE KNOW?
}

\section{Disk fields within a few kpc of the Sun}

\author{
J.L. HAN
}

The Partner Group of MPIfR, National Astronomical Observatories of China, Jia-20 DaTun Rd., Chao Yang District, Beijing 100012, China; E-mail: hjl@bao.ac.cn

\begin{abstract}
The large scale magnetic fields of our Galaxy have been mostly revealed by rotation measures $(R M \mathrm{~s})$ of pulsars and extragalactic radio sources. In the disk of our Galaxy, the average field strength over a few $\mathrm{kpc}$ scale is about $1.8 \mu \mathrm{G}$, while the total field, including the random fields on smaller scales, has a strength of about $5 \mu \mathrm{G}$. The local regular field, if it is part of the large scale field of a bisymmetric form, has a pitch angle of about $-8^{\circ}$. There are at least three, and perhaps five, field reversals from the Norma arm to the outer skirt of our Galaxy.
\end{abstract}

\section{Introduction}

The large scale structure of the magnetic fields in the Milky Way Galaxy is difficult to figure out as it can never be measured completely. Many efforts in the last decade have resulted in a good deal of knowledge, although far from enough. A closelyrelated topic is the measurement of the magnetic fields in nearby spiral galaxies, mainly by multi-wavelength radio polarization observations (see review by Beck $e t$ al., 1996). The regular magnetic field generally has similar orientations parallel to the spiral arms in galactic disks. The magnetic structure could be a superposition of the large scale structure with some local features. The fields were probably generated by dynamos.

I will summarize results on the local field in the vicinity of the Sun, mainly the regular field within a few $\mathrm{kpc}$. I have ignored the measurements of fields in individual clouds which are connected to, but are not part of, the large scale fields. Limited by space here, the magnetic field in the Galactic halo (Han et al., 1997, 1999: HMQ99) and the Galactic center will be discussed in a companion paper (Han, 2002).

There are three key parameters to describe the local field: 1) the field directions, or practically, the pitch angle, $p$, of the field, which is the deviation of the field direction from $l=90^{\circ} ; 2$ ) the field strength of the regular and irregular components, as well as the total field strength, and 3 ) the locations of field reversals. Although we concentrate on the regular field, the irregular random field is the overwhelmingly dominant component in the interstellar medium. 


\section{Pitch Angle}

Using the largest dataset of 7500 stars, Andreasyan and Makarov (1989) concluded that the local field is concentrated in the spiral arms. Reanalyzing the dataset of 7000 stars of Mathewson and Ford (1970), Heiles (1996) obtained for the pitch angle $p=-7.2^{\circ} \pm 4.1^{\circ}$ (see sign definition of the pitch angle in HMQ99) and concluded that the field lines almost follow the spiral pattern. These optical data, all very local and mostly within $1 \mathrm{kpc}$, have been ultimately used already.

Pulsars probably are the only probes of the Galactic magnetic fields on larger scales. Extensive studies became possible only after the $R M$ s of a large sample of pulsars had been measured. Hamilton and Lyne (1987) obtained the $R M \mathrm{~s}$ of 185 pulsars. Rand and Kulkarni (1989: RK89) obtained a field direction of $p=6^{\circ} \pm 4^{\circ}$ from $R M \mathrm{~s}$ of 116 pulsars within $3 \mathrm{kpc}$, a result probably affected by selection effects. Han and Qiao (1994: HQ94) found $p=-8.2^{\circ} \pm 0.5^{\circ}$ from model-fitting to the data of carefully selected pulsars within $3 \mathrm{kpc}$. This result was confirmed by Indrani and Deshpande (1998: ID98) and is consistent with the value from optical data (Heiles, 1996). The projected $R M$ distribution of disk pulsars within $-15^{\circ}<$ $l<15^{\circ}$ shows the transition from positive to negative values almost exactly at the galactic longitude expected from this pitch angle (HMQ99). So, we conclude that the pitch angle of the local regular field is $p=-8^{\circ}$, with a maximum uncertainty of $2^{\circ}$.

\section{Field Strength}

E.M. Berkhuijsen (Figure 5 in Beck et al., 1996), using the deconvolved surface brightness of synchrotron emission from our Galaxy, estimated the total field strength to range from about $10 \mu \mathrm{G}$ at a Galactic radius of $R_{0}=4 \mathrm{kpc}$ to about 5 $\mu \mathrm{G}$ in the vicinity of the Sun.

In fact, the average field strength in our Galaxy can be directly determined from pulsar $D M$ and $R M,\left\langle B_{\|}\right\rangle=1.232 R M / D M$, the best approach to get observational values of the large scale magnetic fields. Manchester (1974) was the first to get the strength of the local regular field, $B=2.2 \pm 0.4 \mu \mathrm{G}$. Thomson and Nelson (1980: TN80) found an average field of $\sim 1 \mu \mathrm{G}$ and a maximum ordered field of $3.5 \mu \mathrm{G}$. Lyne and Smith (1989) used a large dataset of pulsar $R M \mathrm{~s}$ and $D M \mathrm{~s}$ and found the local field strength of about $2 \sim 3 \mu \mathrm{G}$. RK89 found that the average strength of the local field is about $1.6 \pm 0.2 \mu \mathrm{G}$, and the strength of the random field is about $5 \mu \mathrm{G}$. Ohno and Shibata (1993) confirmed this random field strength and they emphasized that the value is independent of the cell size. Rand and Lyne (1994) suggested that the total field strength perhaps reaches $6 \sim 7 \mu \mathrm{G}$ at about $R_{0}=6 \mathrm{kpc}$ and probably increases smoothly towards the Galactic center. HQ94 obtained from pulsar data for the field in the vicinity of the Sun, a regular field strength of about $1.4 \mu \mathrm{G}$, with a maximum regular field strength (in the field 
reversal model) of about $1.8 \pm 0.3 \mu \mathrm{G}$. They also concluded from the residual after model-fitting that the magnetic field energy stored in the random component is 3.7 times that in the regular field, which is consistent with the estimate later made by Zweibel and Heiles (1997) and implies that the total field is about $5 \mu \mathrm{G}$. ID98 confirmed all these results. Both HQ94 and ID98 showed that the regular field is stronger in the interarm region.

All the above results reach a consensus: the strength of the regular field is $1.8 \pm$ $0.3 \mu \mathrm{G}$ and total field is about $5 \mu \mathrm{G}$ locally, but the fields probably become stronger towards the Galactic center.

\section{Field Reversals}

There is no doubt that field reversals exist in our Galaxy. The key points are, 1) how many reversals are there, and 2) where do they occur? Evidence for the nearest field reversal, about $0.2 \mathrm{kpc}$ towards the Galactic center and near the Carina-Sagittarius arm, was first found by TN80 from model-fitting to pulsar $R M$ data and by SimardNormandin and Kronberg (1980: SK80) from model-fitting to the $R M \mathrm{~s}$ of extragalactic radio sources (EGRS). All research thereafter (RK89; Clegg et al., 1992; HQ94; ID98) confirmed this field reversal by similar or different use of more pulsar $R M$ data.

Field reversals, beyond and near the Perseus arm, were first revealed by Lyne and Smith (1989) by comparing the $R M$ values of distant pulsars with those of extragalactic radio sources. The field reversal near the Perseus arm was suggested by the bi-symmetric spiral (BSS) model (SK80; Sofue and Fujimoto, 1983: SF83; HQ94) and was confirmed by Clegg et al. (1992) using newly determined $R M \mathrm{~s}$ of EGRS and by HMQ99 using the $R M \mathrm{~s}$ of pulsars and EGRS. There are some indications (HMQ99) of a further field reversal beyond the Perseus arm at about 15 $\mathrm{kpc}$ from the Sun.

The second field reversal in the inner Galaxy, located near the Crux-Scutum arm, was first shown by $R M$ data in Rand and Lyne (1994). Such a field was also predicted by the BSS model (SF83; HQ94) and later confirmed by more pulsar $R M$ data near $l \sim 327^{\circ}$ (HMQ99). Marginal evidence was found for a field reversal near the Norma arm (HMQ99). More $R M$ data from pulsars, newly discovered in the Parkes multibeam survey will help to figure out the field there.

In summary, there are at least three, perhaps five, field reversals known in our Galaxy. The reversed fields are separated by spiral arms.

\section{Concluding Remarks}

There are three models for the global structure of magnetic fields in the disc of our Galaxy. RK89 and Rand and Lyne (1994) argued that pulsar RMs are consistent 
with a concentric-ring model for the field. This can work for the zero-order approximation, but the pitch angle for the local field of $\sim-8^{\circ}$ does not support this model. Vallee (1996) argued that the field has an axi-symmetric spiral form and no field reversal was allowed beyond $R_{0}=8 \mathrm{kpc}$. The fact of at least one field reversal near or beyond the Perseus arm is not consistent with this model. Early analyses of the $R M$ distribution of extragalactic radio sources (SK80; SF83) suggested that the Galactic magnetic field has a BSS form, in which the field direction reverses from arm to arm. This model seems to be supported by the statistical study of pulsar RMs (HQ94; ID98; HMQ99) and is consistent with the pitch angle and the number of field reversals located over a large range of galactic radii.

\section{Acknowledgements}

I am very grateful to many colleagues, especially, Prof. R.N. Manchester and Prof. G.J. Qiao, for working together with me for a long time to improve the knowledge on the magnetic fields of our Galaxy which I presented here. The research of the author is supported by the National Natural Science Foundation of China, the National Major Project of Fundamental Research, and the Su-Shu Huang Research Foundation of CAS.

\section{References}

Andreasyan, R.R. and Makarov, A.N.: 1989, Astrofiz. 31(2), 247.

Beck, R., Brandenburg, A., Moss, D., Shukurov, A. and Sokoloff, D.: 1996, Annu. Rev. Astron. Astrophys. 34, 155.

Clegg, A.W., Cordes, J.M., Simonetti, J.H. and Kulkarni, S.R.: 1992, Astrophys. J. 386, 143.

Hamilton, P.N. and Lyne, A.G.: 1987, Mon. Not. R. Astron. Soc: 224, 1073.

Han, J.L.: 2002, in Astrophysical Polarized Background, S. Cecchini, S. Cortiglioni, R. Sault and C. Sbarra (eds.), American Institute of Physics, in press.

Han, J.L., Manchester, R.N., Berkhuijsen, E.M. and Beck, R.: 1997, Astron. Astrophys. 322, 98. Han, J.L., Manchester, R.N. and Qiao, G.J.: 1999a, Mon. Not. R. Astron. Soc. 306, 371 (HMQ99).

Han, J.L. and Qiao, G.J.: 1994, Astron. Astrophys. 288, 759 (HQ94).

Heiles, C.: 1996a, Astrophys. J. 462, 316.

Indrani, C. and Deshpande, A.A.: 1998, New Astron. 4, 33 (ID98).

Lyne, A.G. and Smith, F.G.: 1989, Mon. Not. R. Astron. Soc. 237, 533.

Manchester, R.N.: 1974, Astrophys. J. 188, 637.

Mathewson, D.S. and Ford, V.L.: 1970, Mem. R. Astron. Soc. 74, 139.

Ohno, H. and Shibata, S.: 1993, Mon. Not. R. Astron. Soc. 262, 953.

Rand, R.J., and Kulkarni, S.R.: 1989, Astrophys. J. 343, 760 (RK89).

Rand, R.J. and Lyne, A.G.: 1994, Mon. Not. R. Astron. Soc. 268, 497.

Simard-Normandin, M. and Kronberg, P.P.: 1980, Astrophys. J. 242, 74 (SK80).

Sofue, Y. and Fujimoto, M.: 1983, Astrophys. J. 265, 722 (SF83).

Thomson, R.C. and Nelson, A.H.: 1980, Mon. Not. R. Astron. Soc. 191, 863 (TN80).

Vallée, J.P.: 1996, Astron. Astrophys. 308, 433.

Zweibel, E.G. and Heiles, C.: 1997, Nature 385. 131. 